\title{
Estudo qualitativo sobre um programa de educação em saúde bucal ${ }^{\star}$
}

\author{
Qualitative study of an oral health education program
}

\author{
Grasiela Garrett da Silva1, Daniela Lemos Carcereri ${ }^{1}$, Cláudio José Amante ${ }^{1}$
}

\begin{abstract}
Resumo
Introdução: A educação em saúde bucal tem se pautado nos pressupostos da educação bancária (EB). Busca-se superá-la com a educação popular (EP) caracterizada pela construção dialógica. Este estudo qualitativo objetiva compreender a percepção dos envolvidos em um programa de educação em saúde, realizado no estado de Santa Catarina. Métodos: A coleta de dados realizou-se por meio de 4 grupos focais, um em cada município visitado, envolvendo de 3 a 12 professores e gestores em cada grupo. Resultados: Análise de conteúdo de Bardin resultou três categorias: Percepções sobre aspectos metodológicos, conteúdo e participantes. Conclusões: O programa foi percebido como oportunidade de compartilhar experiências sobre saúde bucal, contribuindo com o trabalho da escola. Identificaram-se práticas das duas concepções de educação (EB e EP), denotando traços de transição e dificuldades para concretizar esse câmbio explicadas pelas características do programa e pelo processo histórico no campo da saúde bucal.
\end{abstract}

Palavras-chave: educação em saúde; programas de saúde; saúde bucal.

\begin{abstract}
Introduction: Oral health education has been based on the assumptions of banking education (BE). The aim is to substitute this with popular education (PE) characterized by dialogical construction. This qualitative study aims to understand the perception of those involved in a health education program, conducted in the state of Santa Catarina. Methods: Data collection was carried out with 4 focus groups, one in each city visited, involving 3 to 12 teachers and administrators in each group. Results: Content analysis by Bardin resulted in three categories: Perceptions about methodological aspects, content and participants. Conclusions: The program was perceived as an opportunity to share experiences regarding oral health, including school work. We identified two practices of educational concepts (BE and $\mathrm{PE}$ ), denoting transition traits and difficulty to achieve this exchange explained by the features of the program and the historical processes in the field of oral health.
\end{abstract}

Keywords: health education; health programs; oral health.

\footnotetext{
* A pesquisa é resultado de dissertação de mestrado e obteve financiamento da CAPES - Coordenação de Aperfeiçoamento de Pessoal de Nível Superior (CAPES - PROF) 01/03/2010.

${ }^{1}$ Departamento de Odontologia, Universidade Federal de Santa Catarina (UFSC) - Florianópolis (SC), Brasil.

Trabalho realizado em escolas da rede pública de ensino no estado de Santa Catarina (SC), Brasil.

Endereço para correspondência: Grasiela Garrett da Silva - Programa de Pós-graduação em Odontologia, Centro de Ciências da Saúde, Universidade Federal de Santa Catarina (UFSC), Campus Universitário - Trindade - CEP: 88040-900 - Florianópolis (SC), Brasil - Email: grasielas@yahoo.com

Fonte de financiamento: nenhuma.

Conflito de interesses: nada a declarar.
} 


\section{INTRODUÇÃO}

As ações de educação em saúde são fundamentais como direito de cidadania e melhoria na qualidade de vida. Tradicionalmente, têm se pautado na transmissão de conhecimento visando mudança de hábitos .

Esta pesquisa fundamenta-se na obra de Paulo Freire, apresentando a educação popular (EP) como educação libertadora e dialógica contrapondo-se à educação bancária (EB), entendida como instrumento de dominação ${ }^{2}$ (Figura 1).

A EP valoriza a diversidade e a heterogeneidade e intercomunicação dos grupos sociais, as iniciativas dos educandos e o diálogo entre o saber popular e o saber científico, partindo do saber anterior da população $0^{3-5}$. Os estudos têm se dedicado a entender a forma mais adequada e eficaz de realizar ações educativas preventivas almejando a melhoria na condição bucal da populaçãa ${ }^{1,6,7}, \mathrm{com}$ atividades focadas nas orientações de higiene bucal, na alteração de padrões alimentares inadequados e no encorajamento de retorno frequente ao dentista ${ }^{8}$. Os programas educativos preventivos estudados fundamentam-se em abordagens educativas tradicionais, pautadas na simples transmissão de conhecimentos, sem enfrentar complexidade dos determinantes sociais dos comportamentos e do processo saúde-doença ${ }^{1}$ (Tabela 1).

De acordo com Ferreira et al. ${ }^{5}$ (p. 372),

[...] as práticas de saúde decorrem de experiências contínuas de ensino-aprendizagem e acabam influenciando as decisões a serem tomadas ao longo da existência dos indivíduos, podendo contribuir para diminuir, manter ou elevar o seu nível de saúde.

É desejável que a educação em saúde bucal avance na direção da promoção da saúde criando ambientes que conduzam a saúde, construindo políticas saudáveis, fortalecendo ações comunitárias, desenvolvendo habilidades pessoais e reorientando os serviços de saúde?.

O Prospe é um programa educativo que percorre o Brasil desde 2003, desenvolvendo atividades de educação em saúde em escolas e apresentando um concerto de piano nas praças dos municípios brasileiros ${ }^{11}$. Tem característica itinerante e as ações são desenvolvidas por duas dentistas e um pianista. Com financiamento do governo federal, através da Lei de Incentivo à Cultura, permite a gratuidade das atividades. Contatos e planejamento prévios à chegada nas cidades são realizados com gestores municipais da Educação, Cultura e Saúde; e professores, considerando especificidades locais. Durante o dia, atividades educativas preventivas são realizadas em escolas públicas, e ao anoitecer a população assiste a um concerto de piano na praça central da cidade.

Em 10 anos de atividades por todo o território nacional, tornou-se relevante conhecer e compreender a percepção dos envolvidos, trazendo subsídios para o aperfeiçoamento de programas educativos preventivos em saúde bucal.

\section{MÉTODOS}

O estudo teve abordagem qualitativa, descritiva e exploratória ${ }^{12}$. O estudo foi composto por uma amostra intencional que selecionou seis municípios mais bem representativos do perfil de instituições participantes do Prospe (escolas com menor número de alunos), que fossem de fácil acesso para coleta de dados. Esse critério de inclusão selecionou inicialmente seis municípios catarinenses: Barra Velha, Tijucas, Rio do Sul, Gaspar,

\section{Educação Bancária}

- Tradicional ou convencional

- Transmissão de conhecimento fechada e fragmentada

- Anula poder dos educandos

- Estimula ingenuidade

-Informação é necessária mas não suficiente para escolhas saudáveis

\section{Educação popular}

- Relação dialógica entre educador e educando

- Construção de conhecimento

- Conscientização e autonomia

- Libertadora / problematizadora

- Interação e comunicação

- Escola como espaço educativo e participativo

Figura 1. Concepções educativas em saúde por Paulo Freire $^{2}$

Tabela 1. Características dos programas educativos preventivos em saúde bucal9,10

\begin{tabular}{ccc} 
Concepção educativa & $\begin{array}{c}\text { Educação em saúde bucal tradicional } \\
\text { Educação bancária }\end{array}$ & $\begin{array}{c}\text { Educação em saúde bucal freiriana } \\
\text { Educação popular }\end{array}$ \\
\hline Conteúdo abordado & Doença & $\begin{array}{c}\text { Saúde } \\
\text { Iniciativas }\end{array}$ \\
& $\begin{array}{c}\text { Odontologia sanitária } \\
\text { fundamentada no cuidado } \\
\text { Odontologia simplificada }\end{array}$ & $\begin{array}{c}\text { PROGRAMA DE SAÚDE NA ESCOLA } \\
\text { (PSE) fundamentado na promoção da saúde } \\
\text { e intersetorialidade }\end{array}$ \\
\hline
\end{tabular}


Timbó, Balneário Camboriú. Dentre estes, participaram do estudo os quatro primeiros que apresentaram disponibilidade para participação: Barra Velha, Tijucas, Rio do Sul e Gaspar. A pesquisa foi desenvolvida em quatro escolas de ensino fundamental, nas quais o Prospe foi desenvolvido no estado de Santa Catarina, durante o segundo semestre de 2010.

A coleta de dados foi realizada por um pesquisador, que conduziu os grupos focais e os roteiros de entrevista após instrução e diretrizes do orientador da pesquisa. As etapas de preparo e exercício do pesquisador com o orientador precederam a realização dos grupos focais, bem como a participação em uma disciplina que trata de métodos qualitativos de pesquisa.

Foram realizados 4 grupos focais entre julho e dezembro de 2011. Os grupos focais contaram com número de participantes mínimo de 3 e máximo de 12 . Ao total, 26 pessoas participaram do grupo focal (Figura 2). Os dados foram registrados em gravador de voz digital e diário de campo para anotar aspectos relevantes do processo. Cada grupo focal durou cerca de uma hora e seguiu um roteiro de perguntas semiestruturado.

O participante incluído no grupo focal deveria ter vivenciado as atividades do Prospe em seu município e escola, atuando na organização, apoio logístico e operacional, preparo dos alunos para a atividade ou recepção aos profissionais de saúde. Assim, os grupos focais incluíram secretários municipais, docentes do ensino fundamental, diretores, vice-diretores e coordenadores pedagógicos das escolas.

$\mathrm{O}$ roteiro de entrevista foi do tipo semiestruturado com perguntas abertas, e, a partir das respostas, nortearam-se as perguntas a fim de remeter a análise do objeto a ser estudado. As perguntas foram organizadas de modo a facilitar o diálogo e construídas na forma de perguntas abertas ${ }^{13,14}$. O eixo central das perguntas norteadoras do grupo focal foi a percepção dos participantes sobre o Prospe desenvolvido em seu município por meio do roteiro de entrevista:

1. Primeiramente gostaria que vocês descrevessem o projeto de Educação em Saúde e Música desenvolvido em seu município e escola. Como ele aconteceu?

2. E sobre as atividades desenvolvidas, o que teriam a dizer?
3. Como percebem a realização destas atividades especificamente quanto aos seus realizadores?

4. Qual a percepção de vocês sobre a vivência das pessoas no projeto?

5. Como vocês avaliam a passagem do projeto em seu município e escola?

A pesquisa foi aprovada pelo Comitê de Ética e Pesquisa em Seres Humanos da Universidade Federal de Santa Catarina, sob n. 1.867/2011, conforme resolução vigente. A Resolução 196/96 do Conselho Nacional de Saúde e suas complementares foram observadas e seguidas ao longo de toda a pesquisa.

Após a transcrição das entrevistas, os dados foram analisados pela técnica de Análise de Conteúdo ${ }^{15}$, à luz do referencial teórico de Paulo Freire.

\section{RESULTADOS E DISCUSSÃO}

Da análise dos dados emergiram três categorias, que seguem descritas e ilustradas com relatos relevantes, seguida da discussão, analisando os achados à luz da literatura: percepções sobre aspectos metodológicos, percepções acerca do conteúdo e percepções sobre os participantes.

\section{Percepções sobre aspectos metodológicos}

Os relatos descrevem o Prospe como um programa interativo e dinâmico. A utilização de recursos como materiais coloridos, experiências, fantoches, bonecos e alimentos, e a comunicação artística foram descritas como fortaleza, atraindo a atenção e curiosidade das crianças, envolvendo-as ativamente e deixando-as à vontade para dividir suas experiências e esclarecer dúvidas construindo conhecimento de forma compartilhada e criativa.

[...] achei interessante a dinâmica da apresentação, bem criativo, bem concreto, os materiais que trouxeram, chama atenção das crianças.

$\mathrm{O}$ aspecto lúdico, adequação da linguagem à faixa etária e recursos adequados são condições para conquistar atenção das crianças e interagir construtivamente, conferindo aos programas educativo-preventivos dinamicidade, condições fundamentais para o trabalho com crianças ${ }^{16}$.

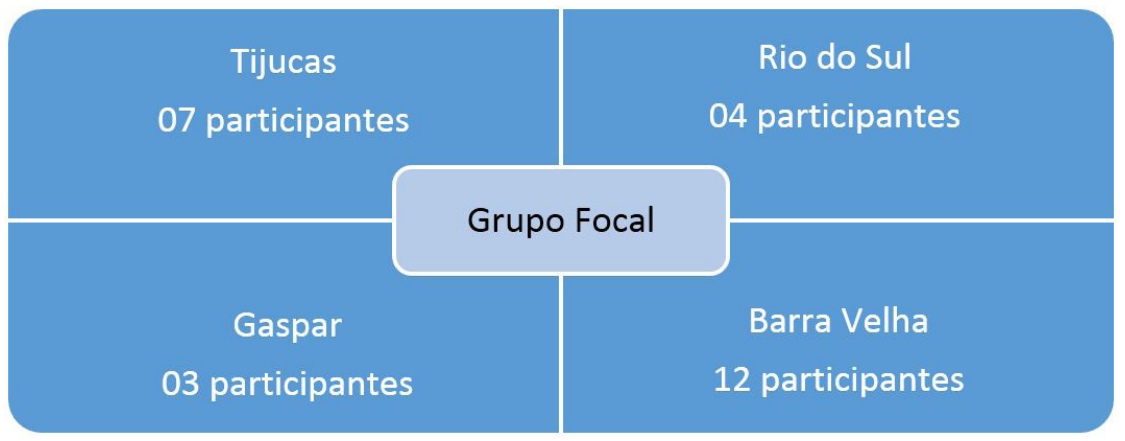

Figura 2. Grupos focais (cidades e número de participantes) 
No planejamento, o Prospe contata previamente as escolas convidando a comunidade escolar para elaboração das atividades, agregando a visão pedagógica especializada.

Esse envolvimento com as profissionais de saúde conferiu credibilidade ao abordar os temas, permitindo aprendizado das crianças e compartilhamento da vivência com os seus pais e familiares, retornando às questões em oportunidades posteriores na escola.

Eu vi bastante envolvimento do grupo, as crianças também participaram no dia, fizeram bastante questionamento, foi gratificante ver como se envolveram ...

As práticas educativas em saúde foram identificadas como instrumento de mudança de comportamento, por meio do conhecimento técnico-científico, adquirindo hábitos saudáveis para a prevenção das doenças bucais ${ }^{17}$. As tendências encontradas nos programas de saúde bucal para escolares consideram a dimensão educativa fundamentando-a na prática de transmissão de conhecimentos, sem encorajar os escolares à dialogicidade e autonomia no cuidado à saúde ${ }^{18}$.

É preciso substituir modelos ancorados em práticas de comunicação unidirecional, dogmática e autoritária pela discussão e reflexão, problematizando temas da saúde bucal e considerando a complexidade dos determinantes sociais dos comportamentos e do processo saúde-doença ${ }^{1-8}$.

Ao trabalhar com base nesse enfoque, o profissional interage com os participantes do grupo, estimulando sua consciência crítica e o exercício da sua autonomia frente às decisões de saúde no âmbito individual e coletivo ${ }^{16}$.

Sobre a frequência e continuidade, relataram que o fato do projeto ser pontual é um limitador ao desenvolvimento de um trabalho educativo. Destacaram a importância do retorno do Prospe indicando anseio de atenção à saúde de forma contínua.

[...] se já foi interessante naquele momento, imagine se você der continuidade, seria bem interessante.

Os relatos apontaram ser esta uma constante encontrada nas instituições, considerando que os programas executam suas atividades durante um determinado período e as interrompem por falta de profissionais, de material, de incentivo. Sugere-se a parceria com as Equipes de Saúde Bucal da Estratégia Saúde da Família (ESB), garantindo continuidade e frequência das atividades, com a participação integrada dos envolvidos.

A escola tem seu papel de instrução e reforço de condutas sobre saúde bucal, como a escovação supervisionada, porém a parceria com profissionais de saúde é importante, visto que este não é o objeto primeiro de sua atividade ${ }^{19}$. A motivação, com sessões de reforço contínuas, deve assegurar o sucesso dos trabalhos educativo-preventivos ${ }^{20}$.

Os municípios visitados pelo Prospe seguem a Política Nacional de Saúde Bucal ${ }^{21}$, buscam trabalhar na lógica da Estratégia Saúde da Família (ESF) e atender os pressupostos de adscrição populacional, longitudinalidade do cuidado e articulação intersetorial de modo especial por meio do Programa Saúde na Escola (PSE) ${ }^{22}$ (Tabela 2).

As escolas visitadas pelo Prospe foram indicadas pelas Secretarias Municipais de Educação e Saúde considerando a oferta de ações educativas de saúde bucal. Após a passagem do Prospe, percebeu-se o empenho dos pais para o atendimento odontológico para seus filhos.

A presença do cirurgião-dentista (CD) confere credibilidade para realização deste tipo de atividade.

[...] é diferente de chegar outra pessoa com a roupa preparada, adequada, formada para aquilo, dá uma outra ênfase. Isto causa muita diferença para a criança, o impacto é muito maior.

É preciso considerar o papel CD no processo de trabalho em equipe, proposto pelo Sistema Único de Saúde (SUS). Dentre suas atribuições, desempenha funções clínicas, educativas, preventivas e epidemiológicas, atua como coordenador de Auxiliares em Saúde Bucal (ASB) e Técnicos em Saúde Bucal $(\mathrm{TSB})^{9}$. Sua presença na unidade de saúde é fundamental no processo de trabalho, e, nas escolas, não há necessidade de estar sempre presente, oportunizando aos profissionais auxiliares trabalhar eficientemente. O CD articulador e coordenador coordena e conhece o trabalho e as necessidades da população, vincula-se à escola e confere credibilidade aos parceiros, estando nas escolas em momentos estratégicos. Um projeto educativo longitudinal deve ser potente para promover a saúde bucal no ambiente escolar de forma a facilitar o acesso às escolhas saudáveis. Isso implica em incorporar o conteúdo de saúde bucal em diferentes estratégias escolares, integrando-as ao currículo e ao calendário escolar 9 .

Tabela 2. Adesão dos municípios catarinenses ao Programa Saúde na Escola (PSE) em 2014

\begin{tabular}{lcccc} 
Localidade & $\begin{array}{c}\text { Total de } \\
\text { escolas }^{*}\end{array}$ & $\begin{array}{c}\text { Total de educandos } \\
\text { pactuados }\end{array}$ & $\begin{array}{c}\text { Total de escolas } \\
\text { pactuadas }\end{array}$ & $\begin{array}{c}\text { Total de } \\
\text { equipes PSE }\end{array}$ \\
Estado de Santa Catarina & 5.181 & 547.329 & 2.703 & 1.242 \\
Município de Barra Velha & 19 & 313 & 3 & 3 \\
Município de Gaspar & 36 & 5.588 & 25 & 11 \\
Município de Rio de Sul & 48 & 10.231 & 52 & 15 \\
Município de Tijucas & 28 & 4.794 & 17 & 7 \\
\hline
\end{tabular}

${ }^{*}$ Fonte: Censo Escolar/ASEST/SED/SC ${ }^{22}$ 
Os relatos informam que o CD educador, idealmente, é o profissional que direcione atenção às crianças, compartilhando cuidados de higiene bucal. Os CDs parecem ocupar um lugar de destaque como veículo de informação ${ }^{1}$, devendo a equipe de saúde bucal orientar esse trabalho ${ }^{9}$ e os CDs trabalharem para que a prevenção da cárie dentária e periodontopatias por meio da higienização seja instituída nos consultórios, nas escolas e nas unidades de saúde, apoiada e motivada constantemente ${ }^{20}$.

Percebe-se a hegemonia da concepção de educação tradicional, centrado no CD. É oportuno mudar o estilo de pensamento; aprender a trabalhar com a educação popular, considerando os saberes da comunidade.

A intersetorialidade não foi percebida pelos envolvidos, conforme previsto pelo programa. Embora envolvendo as Secretarias Municipais de Cultura, Educação e Saúde, foi percebido como um programa da Secretaria de Educação, em virtude da realização em ambiente escolar.

Os profissionais de saúde orientam suas atividades nos preceitos de integralidade e intersetorialidade, entretanto é um desafio constante, pois requer a conscientização e vontade coletiva para sua execução.

Foi ressaltada a importância do preparo prévio das escolas para recebimento do Prospe. A vinda do programa motivou os professores a trabalharem o conteúdo de saúde bucal preparando as crianças para as atividades. Adicionalmente, proporcionou discussão em sala de aula após a passagem do programa.

[...] achei que foi ótimo, foi bem comentado, deu para aproveitar em sala de aula, para trabalhar naquele dia, naquela semana, porque eles gostaram bastante ...

O envolvimento das escolas para receber o programa, com a inclusão do conteúdo de saúde bucal em suas atividades, atestou o interesse e valorização que as escolas destinam aos cuidados com a saúde.

Sobre o financiamento do programa:

[...] no início eu logo perguntei qual seria o custo, porque é difícil você receber um projeto desse nível, na escola, que não tenha custo algum $[\ldots]$

O financiamento foi percebido como um ponto importante e facilitador para desenvolvimento do Prospe, pois essas iniciativas requerem investimento tal qual ocorre nos projetos desenvolvidos no âmbito da ESF.

\section{Percepções acerca do conteúdo}

Os envolvidos perceberam o projeto como multitemático, identificando conteúdos sobre promoção da saúde, prevenção de doenças, cidadania, arte, música, importância do cuidado com a saúde e importância do $\mathrm{CD}$, adequados à faixa etária e integrados a atividades práticas.

[...] a parte prática foi fantástica, aqueles escovódromos no pátio, e eles participando, aí fechou com chave de ouro.
[...] foi um projeto ímpar, nunca tínhamos tido nada parecido e de nenhuma outra área $[. .$.

[...] começou pelo lanche ... mudou completamente, todo dia eles estavam vindo com fruta.

$\mathrm{Na}$ prevenção "se radicam as possibilidades práticas da educação e da promoção da saúde, da proteção específica, do diagnóstico precoce, do tratamento e da limitação do dano e da reabilitação, em escalas ou níveis crescentes"23.

A importância da higiene bucal, orientações sobre escovação, alimentação, desenvolvimento da doença cárie e hábitos saudáveis foram destacados pelos envolvidos, merecendo permanente inclusão em programas de saúde pública pela importância no âmbito da saúde bucal.

Adicionalmente, o resgate de conteúdos sobre saúde bucal com frequência, enfatizando a importância do cuidado e motivando as crianças constantemente ${ }^{20}$.

[...] alguns dias depois, as crianças se preocupam, mas depois cai no esquecimento, tem que ir resgatando para que eles tenham realmente higiene bucal.

A vivência e compreensão do conteúdo proporcionaram reflexão sobre sua própria saúde, incentivando boas práticas de autocuidado. Isso se reflete na motivação em procurar o profissional da saúde para orientações e tratamentos específicos para suas necessidades.

De acordo com os parâmetros do Ministério da Saúde, o conteúdo para as ações educativas coletivas contempla as principais doenças bucais, como se manifestam e são prevenidas; a importância do autocuidado, da higiene bucal, da escovação com dentifrício fluoretado e o uso do fio dental; os cuidados a serem tomados para evitar a fluorose; as orientações gerais sobre alimentação saudável; a orientação para autoexame da boca; os cuidados imediatos após traumatismo dentário; a prevenção à exposição ao sol sem proteção; e a prevenção ao uso de álcool e fumo?.

A população valoriza iniciativas pautadas na promoção de saúde e resolutividade de seus problemas, devendo a educação em saúde focalizar nas ações comunitárias factíveis, considerando especificidades locais. Considerando os benefícios do trabalho em grupo, pode-se pensar na implementação de programas educativos voltados para os professores, a fim de abordarem esse tema em sala de aula ${ }^{24}$.

\section{Percepções sobre os participantes}

Os relatos apontam o interesse, adesão e envolvimento dos participantes em todos os momentos da passagem do programa por seu município, desde a organização à realização das atividades.

A atuação da escola, da direção e dos professores foi importante nas atividades educativas e a participação das crianças reflete o cuidado dos professores e gestores sobre sua saúde. 
Os envolvidos identificam que os pais poderiam estar mais presentes na educação e saúde, a fim de serem o exemplo para seus filhos.

[...] a criança aprende com o exemplo, e tendo o pai e a mãe em casa, é um exemplo que a criança tem.

[...] seria interessante envolver os pais neste projeto, para eles ajudarem os filhos, para eles darem o exemplo [...] seria um passo além para o projeto ...

Pensando na importância da presença dos pais nas atividades, os programas podem considerar a disponibilidade de participação dos pais, melhorando a condição de saúde de todos. Compreende-se como uma limitação do estudo não ter ouvido os pais para conhecer suas percepções.

O envolvimento e planejamento conjunto das atividades foi fundamental para alcançar resultados:

[...] isto foi amplamente divulgado, os alunos fizeram cartazes, trabalharam a respeito e nós falamos sobre escovação, sobre necessidade da higiene bucal.

As ações educativas realizadas na escola mostram-se efetivas na melhora das condições de saúde bucal e de hábitos, sendo importante o processo de capacitação e o planejamento conjunto das ações entre os profissionais da escola e os profissionais de saúde ${ }^{25}$.

A educação promotora em saúde tem sido destacada como emancipadora do sujeito, permitindo que ele transforme sua realidade e estimule experiências para o desenvolvimento de melhores condições de vida e promoção da saúde. As práticas educativas em saúde facilitam a troca de conhecimento e experiências entre os membros da comunidade e profissionais, com isso fornecendo liberdade a momentos de debates reflexivos que possibilitam às pessoas um aprendizado significativo ${ }^{5}$.

\section{CONSIDERAÇÕES FINAIS}

O estudo foi capaz de desvelar as percepções dos participantes em relação às ações desenvolvidas pelo Prospe.

Os relatos enfocaram o método utilizado, o conteúdo abordado e os sujeitos envolvidos, apontando que o Prospe foi percebido como oportunidade de compartilhar experiências sobre saúde bucal e contribuição ao trabalho da escola.

Os participantes valorizaram o envolvimento dos responsáveis com o programa, o cuidado em adequar o projeto ao contexto e a interação com os saberes da comunidade. Destacaram como positiva a participação de professores e gestores durante a organização das atividades, e das crianças durante o desenvolvimento do programa. O Prospe vivenciado foi inovador considerando os programas já recebidos.

O estudo traz subsídios à prática de educação em saúde bucal pautada nos pressupostos epistemológicos da educação popular em saúde.

Sugere-se o desenvolvimento de pesquisas que estudem a percepção dos pais sobre sua participação nas atividades propostas pelas escolas.

\section{REFERÊNCIAS}

1. Lemkuhl I, Souza MVC, Cascaes AM, Bastos JL. A efetividade das intervenções educativas em saúde bucal: revisão de literatura. Cad Saúde Colet. 2015;23(3):336-46. http://dx.doi.org/10.1590/1414-462X201400030104.

2. Freire P. Pedagogia do oprimido. 50 ed. Rio de Janeiro: Paz e Terra, 2011.

3. Stotz E. Enfoques sobre educação popular e saúde. In: Brasil. Ministério da Saúde. Secretaria de Gestão Estratégica e Participativa. Departamento de Apoio à Gestão Participativa. Caderno de Educação Popular e Saúde. Brasília: Ministério da Saúde; 2007. p. 46-57. (Série B. Textos Básicos de Saúde).

4. Vasconcelos EM. Educação popular e atenção à saúde da família. 4 ed. São Paulo: Hucitec, 2008.

5. Ferreira VF, Rocha GOR, Lopes MMB, Santos MS, Miranda AS. Educação em saúde e cidadania: revisão integrativa. Trab Educ Saúde. 2014;12(2):36378. http://dx.doi.org/10.1590/S1981-77462014000200009.

6. Silva CMC, Meneghim MC, Pereira AC, Mialhe FL. Educação em saúde: uma reflexão histórica de suas práticas. Ciênc Saúde Coletiva. 2010;15(5):253950. http://dx.doi.org/10.1590/S1413-81232010000500028.

7. Kusma SZ, Moyses ST, Moyses SJ. Avaliação de Efetividade de Estratégias de Promoção de Saúde Bucal: ferramenta de avaliação. Curitiba: PUCPR, 2010.

8. Cervera DPP, Parreira BDM, Goulart BF. Educação em saúde: percepção dos enfermeiros da atenção básica em Uberaba (MG). Cien Saude Colet.

2011;16(Supl 1):1547-54. PMid:21503506. http://dx.doi.org/10.1590/ S1413-81232011000700090.

9. Brasil. Ministério da Saúde. Secretaria de Atenção à Saúde. Departamento de Atenção Básica. Cadernos de Atenção Básica. Brasília: Ministério da Saúde; 2008.92 p. no. 17.

10. Brasil. Ministério da Saúde. Secretaria de Atenção à Saúde. Departamento de Atenção Básica. Cadernos de Atenção Básica: saúde na escola. Brasília: Ministério da Saúde; 2009. 100 p.

11. Lima AM. Um Piano pela Estrada [Internet]. Florianópolis: Aml Cultural Ltda; c2008. Projeto [citado em 2015 dez 18]. Disponível em: http://www. arthurmoreiralima.com.br/site/pianopelaestrada.htm

12. Deslauriers JP, Kerisit M. O delineamento de pesquisa qualitativa. In: Poupart J, Deslauriers JP, Groulx LH, Laperrière A, Mayer R, Pires AP. A pesquisa qualitativa: enfoques epistemológicos e metodológicos. Petrópolis: Vozes, 2008. p. 127-53.

13. Minayo MCS. Trabalho de campo: contexto de observação, interação e descoberta. In: Deslandes SF, Gomes R, Minayo MCS, organizadores. Pesquisa social: teoria, método e criatividade. Petrópolis: Vozes, 2012. p. 61-77.

14. Minayo MCS. Análise qualitativa: teoria, passos e fidedignidade. Ciênc Saúde Coletiva. 2012;17(3):621-26. http://dx.doi.org/10.1590/S141381232012000300007 
15. Bardin L. Análise de conteúdo. 4 ed. Lisboa: Editora 70; 2009.

16. Munguba MCS. Educação na saúde: sobreposição de saberes ou interface? [Internet]. RBPS. 2010;23(4):295-96. [citado em 2015 dez 18]. Disponível em: http://ojs.unifor.br/index.php/RBPS/article/viewFile/2029/2324

17. Mialhe FL, Silva CMC. A educação em saúde e suas representações entre alunos de um curso de odontologia. Ciênc Saúde Coletiva. 2011;16(1):155561. http://dx.doi.org/10.1590/S1413-81232011000700091.

18. Pauleto ARC, Pereira MLT, Goldfarb CE. Saúde bucal: uma revisão crítica sobre programações educativas para escolares. Ciênc Saúde Coletiva. 2004;9(1):121-30. http://dx.doi.org/10.1590/S1413-81232004000100012.

19. Gubert FA, Santos ACL, Aragão KA, Pereira DCR, Vieira NFC, Pinheiro PNC. Tecnologias educativas no contexto escolar: estratégia de educação em saúde em escola pública de Fortaleza CE [Internet]. Rev Eletr Enf. 2009;11(1):165-72. [citado em 2015 dez 18]. Disponível em: http://www. fen.ufg.br/revista/v11/n1/v11n1a21.htm

20. Toassi RFC, Cauhy PP. Motivação no controle do biofilme dental e sangramento gengival em escolares. Rev Saúde Pública. 2002;36(5):634-37. http://dx.doi.org/10.1590/S0034-89102002000600015.
21. Brasil. Ministério da Saúde. Secretaria de Atenção à Saúde. Departamento de Atenção Básica. Diretrizes da Política Nacional de Saúde Bucal. Brasília: Ministério da Saúde, 2004. 16 p.

22. Santa Catarina. Secretaria de Estado da Saúde de Santa Catarina (SES-SC). Adesões ao PSE SC em 2014. Florianópolis: SES-SC, 2015.

23. Botazzo C. Sobre a Atenção Básica. Os cuidados primários de saúde no contexto da Reforma Sanitária Brasileira. In: Botazzo C, Oliveira MA organizadores. Atenção básica no Sistema Único de Saúde: abordagens interdisciplinares para os serviços de saúde bucal. São Paulo: Páginas \& Letras Editora e Gráfica, 2008. p. 1-12.

24. Tanaka C, Borghi WMMC, Moimaz SAS, Saliba NA, Garbin CAS. Análise do conteúdo sobre saúde bucal no material didático da disciplina de ciências utilizado em escolas de ensino fundamental. Rev Odontol UNESP. 2008;37(2):103-7.

25. Turrioni APS, Salomão FGD, Monti JFC, Vazquez FL, Cortellazzi KL, Pereira AC. Avaliação das ações de educação na saúde bucal de adolescentes dentro da Estratégia de Saúde da Família. Ciênc Saúde Coletiva. 2012;17(7):184148. http://dx.doi.org/10.1590/S1413-81232012000700023.

Recebido em: Set 16, 2016 Aprovado em: Mar 10, 2017 